\title{
Oxide muonics: A new compendium
}

\author{
S.F.J. Cox ${ }^{\mathrm{a}, \mathrm{b}, *}$, J.S. Lord ${ }^{\mathrm{a}}$, S.P. Cottrell ${ }^{\mathrm{a}}$, J.M. Gil ${ }^{\mathrm{c}}$, H.V. Alberto ${ }^{\mathrm{c}}$, J. Piroto Duarte ${ }^{\mathrm{c}}$, \\ R.C. Vilão ${ }^{c}$, D.J. Keeble ${ }^{d}$, E.A. Davis ${ }^{\text {e }}$, A. Keren ${ }^{f}$, R. Scheuermann ${ }^{g}$, A. Stoykov ${ }^{g}$, \\ M. Charlton ${ }^{\text {h }}$, D.P. van der Werf ${ }^{\text {h }}$, J. Gavartin ${ }^{\mathrm{b}}$ \\ a ISIS Facility, Rutherford Appleton Laboratory, Chilton OX11 0QX, UK \\ ${ }^{\mathrm{b}}$ Condensed Matter and Materials Physics, University College London, WC1E 6BT, UK \\ ${ }^{\mathrm{c}}$ Physics Department, University of Coimbra, P-3004-516 Portugal \\ ${ }^{\mathrm{d}}$ Electronic Engineering and Physics Division, University of Dundee, DD1 4HN, UK \\ ${ }^{\mathrm{e}}$ Materials Science and Metallurgy Department, University of Cambridge, CB2 3QZ, UK \\ ${ }^{\mathrm{f}}$ Physics Department, Technion, 32000 Haifa Israel \\ ${ }^{\mathrm{g}}$ Paul Scherrer Institute, CH-5232 Villigen, PSI Switzerland \\ ${ }^{\mathrm{h}}$ Physics Department, University of Wales, Swansea SA2 8PP, UK
}

\begin{abstract}
A new survey of muonium states brings the total of binary non-magnetic oxides studied to 30, with normal muonium-the interstitially trapped atomic state - found in 15 of these. The number of shallow-donor states of the type known in ZnO now also totals 15 , but there are hints of several others. Tantalizingly, the shallow-donor and deep-atomic states are found to coexist in several of the candidate highpermittivity dielectrics. Highly anisotropic states, resembling anomalous muonium in semiconductors and including examples of muonium trapped at oxygen vacancies, complete a spectrum of hyperfine parameters covering five powers of ten. Effective ionization temperatures range from $10 \mathrm{~K}$ for shallow to over $1000 \mathrm{~K}$ for deep states, with corresponding activation energies between several meV and several $\mathrm{eV}$. The oxide band gap emerges as a parameter controlling the systematics of the deep-to-shallow transition for muonium and, by inference, monatomic hydrogen.
\end{abstract}

(C) 2005 Elsevier B.V. All rights reserved.

PACS: 77.84.Bw; 71.55.Gs; 31.30.Gs; 36.10.Dr; 76.75.+i

Keywords: Muonium; Hydrogen doping

\section{Introduction}

Since the earliest spectroscopic studies of muonium in quartz, and in a relatively small number of other wide-gap non-magnetic oxides, the use of muonium as a model for hydrogen has taken on a new importance. This follows $\mu \mathrm{SR}$ confirmation that hydrogen forms a shallow-donor state in $\mathrm{ZnO}$; that is, its electron wave function does not retain atomic character but delocalizes into conduction-band states. The question arises as to whether hydrogen can similarly act as an n-type

\footnotetext{
${ }^{*}$ Corresponding author. ISIS Facility, Rutherford Appleton Laboratory, Chilton OX11 0QX, UK. Fax: + 441235445720.
}

dopant and induce electronic conductivity in other oxides. A particular concern is that it might do so in those high-permittivity materials such as $\mathrm{HfO}_{2}$ and $\mathrm{ZrO}_{2}$ that are destined to replace $\mathrm{SiO}_{2}$ as nano-scale gate dielectrics. We have therefore undertaken a new survey of muonium states in a wide selection of oxides, both semiconducting and dielectric. We use the term muonics to describe the use of muonium data as a model for the electronic structure and electrical activity of hydrogen in the dilute monatomic limit. Comparable ESR data for $\mathrm{H}$ in oxides is sparse, but fortunately include the trapped atoms in $\mathrm{SiO}_{2}$ and $\mathrm{Li}_{2} \mathrm{O}$, as well as the donor in $\mathrm{ZnO}$, thereby validating the muonics principle both for deep and shallow states. 

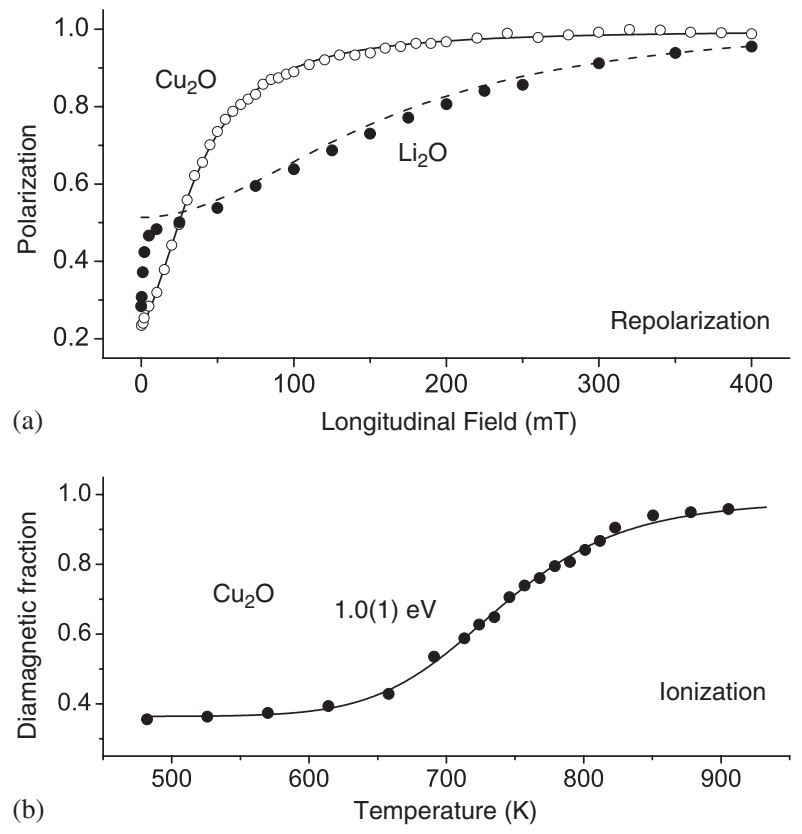

Fig. 1. Repolarization curves (a) showing the different hyperfine constants in $\mathrm{Cu}_{2} \mathrm{O}$ and $\mathrm{Li}_{2} \mathrm{O}$, as well as indications of nuclear couplings. The expectation for free muonium (dashed curve) compares well with the $\mathrm{Li}_{2} \mathrm{O}$ data but the $\mathrm{Cu}_{2} \mathrm{O}$ data require a model fit (solid line). Growth of the diamagnetic $\left(\mathrm{Mu}^{+}\right.$or $\left.\mathrm{Mu}^{-}\right)$fraction at high temperature, as paramagnetic muonium disappears, is shown for $\mathrm{Cu}_{2} \mathrm{O}$ in (b) and fitted for an effective ionization energy.

\section{Normal muonium}

Much studied in quartz and ice, the interstitially trapped atom known as normal muonium was also known to early $\mu$ SR studies in $\mathrm{Al}_{2} \mathrm{O}_{3}, \mathrm{BeO}, \mathrm{CaO}$ and $\mathrm{MgO}$ [1-6]. Using the longitudinal-field method of hyperfine decoupling or repolarization (as in the first studies of $\mathrm{Al}_{2} \mathrm{O}_{3}$ [1]), we have also detected normal muonium in polycrystalline samples of $\mathrm{Bi}_{2} \mathrm{O}_{3}, \mathrm{Cu}_{2} \mathrm{O}, \mathrm{HfO}_{2}, \mathrm{La}_{2} \mathrm{O}_{3}, \mathrm{Li}_{2} \mathrm{O}, \mathrm{Sb}_{2} \mathrm{O}_{3}, \mathrm{SrO}, \mathrm{Y}_{2} \mathrm{O}_{3}$, $\mathrm{ZrO}_{2}$ and YSZ. ${ }^{1}$ Examples are shown in Fig. 1(a). The hyperfine constants are mostly close to the free-atom value, with the notable exception of $\mathrm{Cu}_{2} \mathrm{O}$, where a reduction to $30 \%$ is reminiscent of muonium in the cuprous halides [9].

Normal muonium is invariably stable at room temperature; it is still detectable at $1300 \mathrm{~K}$ in quartz [10] but more usually disappears in the intervening range. Fig. 1(b) shows an example of the concomitant growth of diamagnetic fraction, fitted with an effective ionization energy which, for $\mathrm{Cu}_{2} \mathrm{O}$ as for $\mathrm{Y}_{2} \mathrm{O}_{3}$, places an electrically active level near midgap. It is tempting to assign this to a deep acceptor function (i.e. to hole-ionization, $\mathrm{Mu}^{0} \rightarrow \mathrm{Mu}^{-}+\mathrm{h}$ ) but motional narrowing precludes site and charge-state identification for the high-T state. Two activation steps are necessary for $\mathrm{ZrO}_{2}$ and $\mathrm{HfO}_{2}$ (with both activation

\footnotetext{
${ }^{1}$ In our view, it is muonium formation rather than unconventional magnetism that is responsible for fast depolarization [7] in $\mathrm{Bi}_{2} \mathrm{O}_{3}$. Normal muonium in $\mathrm{ZrO}_{2}$ is also reported in PSI data [8]. YSZ = yttria-stabilized cubic zirconia.
}
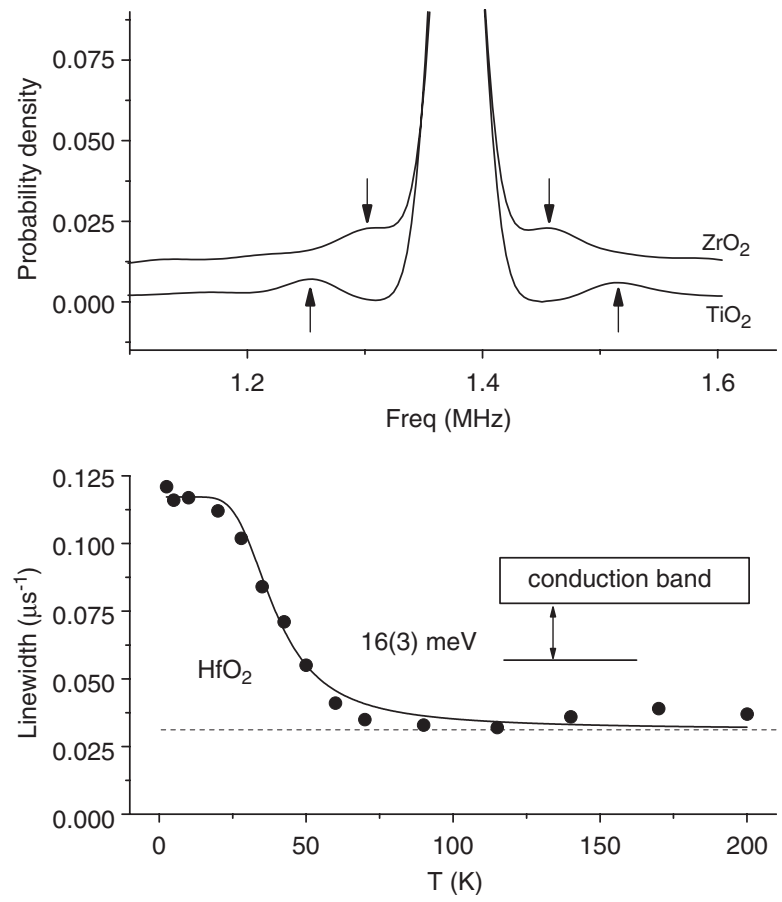

Fig. 2. $\mu \mathrm{SR}$ spectra (a) showing shallow-donor hyperfine satellites (arrowed) in $\mathrm{TiO}_{2}$ and (less well resolved) in $\mathrm{c}-\mathrm{ZrO}_{2}$ (YSZ). The temperature dependence of overall linewidth is shown for $\mathrm{HfO}_{2}$ in (b), where the dashed line is the estimated nuclear broadening and the fitted dissociation energy provides a guide to the donor depth.

energies less than half the band gap) and it may be that conversion to both $\mathrm{Mu}^{+}$and $\mathrm{Mu}^{-}$are important.

\section{Shallow-donor muonium}

In the early studies, it was puzzling why muonium did not seem to be formed in all other wide-gap oxides. It may be that shallow-donor states of the type now known in $\mathrm{ZnO}[11,12]$ were overlooked: the weak binding energy and very low hyperfine constants require a careful search for broadening or splitting of the Larmor precession spectrum at cryogenic temperatures. Thus $\mathrm{TiO}_{2}$ was originally thought to show only a diamagnetic muon state [4] and the significance of a low-T broadening of its polycrystalline spectrum in more recent ISIS data [13] was not at first recognized. For rutile- $\mathrm{TiO}_{2}$, this broadening is now seen to resolve itself into hyperfine satellites characteristic of a shallow-donor state, as in the single-crystal ISIS data of Fig. 2(a) as well as in independent data from KEK [14]. Similar spectra are seen clearly even for polycrystalline $\mathrm{CdO}$ and $\mathrm{BaO}$. The shallow-donor hyperfine constants are typically 4 or 5 orders of magnitude lower than for atomic muonium. Varying degrees of anisotropy in the shallow centres suggest that polaronic rather than hydrogenic models may be appropriate [15]. For $\mathrm{ZnO}$, doubleresonance measurements of $g$-factor nonetheless confirm the conduction-band character of the diffuse electron orbital [16]. 
Even when the characteristic satellites are not resolved (whether due to short spin-state lifetimes or, in polycrystalline spectra, anisotropy), formation of shallow-donor muonium states can still be inferred from the temperature dependence of overall linewidth. This is so for materials such as $\mathrm{CeO}_{2}, \mathrm{HfO}_{2}, \mathrm{SnO}_{2}$, anatase- $\mathrm{TiO}_{2}, \mathrm{WO}_{3}$ and $\mathrm{ZrO}_{2}$ : Fig. 2(b) shows ISIS data for $\mathrm{HfO}_{2}$ as an example, where the paramagnetic broadening is visible over and above the estimated nuclear contribution. For materials with stronger nuclear magnetism, this separation is more difficult, but there are hints of such states in $\mathrm{La}_{2} \mathrm{O}_{3}, \mathrm{Nb}_{2} \mathrm{O}_{5}$ and $\mathrm{Ta}_{2} \mathrm{O}_{5}$. An intriguing reaction sequence in $\mathrm{Ag}_{2} \mathrm{O}$, elucidated by $\mathrm{RF}$ and level-crossing resonance in an accompanying paper [17], shows a final state that also appears to qualify as a shallow donor.

\section{Anomalous muonium}

The oxides also show several examples of highly anisotropic muonium states with hyperfine parameters reminiscent of anomalous muonium $\left(\mathrm{Mu}^{*}\right)$ in tetrahedral semiconductors. Muonium in $\mathrm{HgO}$ shows a contact term of $15 \mathrm{MHz}$ and a dipolar term of $5 \mathrm{MHz}$, giving rise to a striking level-crossing resonance at $55 \mathrm{mT}$ [18]; motional effects described in an accompanying paper suggest a muon site not at the bond-centre but antibonding to oxygen [19]. The parameters imply a reasonably compact electronic orbital, i.e. a deep state. Those for the precursor to the shallow donor in $\mathrm{Ag}_{2} \mathrm{O}$ are not dissimilar [17]. $\mathrm{CeO}_{2}$ presents an intriguing case with a contact term of just $0.8 \mathrm{MHz}$ and a relatively large dipolar term of $17 \mathrm{MHz}$ [15].
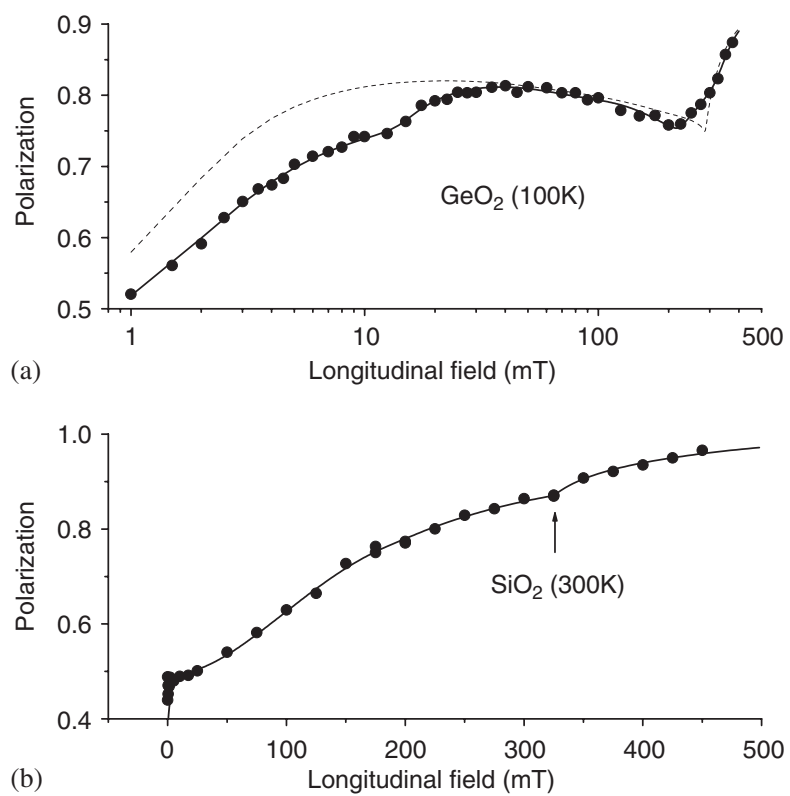

Fig. 3. Repolarization and level-crossing resonance data for (a) $\mathrm{GeO}_{2}$ and (b) $\mathrm{SiO}_{2}$ - both polycrystalline samples. The dashed curve in (a) is a simulation for a single centre with axial symmetry, the solid line a fit with full anisotropy and precursor states; note log scale. The fitted line and linear scale in (b) show the vacancy-complex resonance superimposed on normal-muonium repolarization.
Inspired by reports of unassigned frequencies in the $\mu \mathrm{SR}$ spectrum of $\mathrm{GeO}_{2}$ [20], our repolarization studies of this material (Fig. 3a) reveal a centre with a contact term of $56 \mathrm{MHz}$ and a dipolar term of $51 \mathrm{MHz}$, with opposite signs. As well as resembling values for $\mathrm{Mu}^{*}$ in elemental Ge, they scale closely with ESR parameters for the $E_{4}^{\prime}$ centre in $\mathrm{SiO}_{2}$. This latter is assigned to $\mathrm{H}$ trapped at an oxygen vacancy: the resultant bridging site between two cations explains the resemblance to bond-centred muonium in tetrahedral semiconductors. Fig. 3(b) shows that such a muonium-vacancy complex ( $A=76, D=24 \mathrm{MHz}$ ) appears also to coexist with normal muonium in $\mathrm{SiO}_{2}{ }^{2}$

\section{Band gap correlation, systematics and implications for doping}

The shallow-donor muonium states all dissociate below about $100 \mathrm{~K}$ (in $\mathrm{BaO}$ and rutile- $\mathrm{TiO}_{2}$ as low as $10 \mathrm{~K}$ ), with effective ionization energies varying from several $\mathrm{meV}$ to several tens of meV. The anomalous muonia show varying degrees of thermal stability and probably act as deep donors, dissociating below room temperature in $\mathrm{HgO}$ $(125-225 \mathrm{~K}, 0.15-0.3 \mathrm{eV})$ but above room temperature for the vacancy-complex in $\mathrm{GeO}_{2}(500-700 \mathrm{~K}, 0.9 \mathrm{eV})$. Normal muonium disappears only well above room temperature, with a range of activation energies between a few hundred $\mathrm{meV}$ and several $\mathrm{eV}$, often in two steps suggesting independent processes. Further details of all the measurements and results, the comparison with current theoretical predictions and a discussion of the high- $\mathrm{T}$ behaviour, are given elsewhere [15].

Two outcomes of the new survey are particularly striking. One is the coexistence of deep and shallow states in certain oxides - something that has not previously been addressed theoretically. The other is a correlation between the occurrence of deep and shallow states with host band gap. Only normal (atomic) muonium is found in oxides with band gaps above about $7 \mathrm{eV}\left(\mathrm{BeO}, \mathrm{Al}_{2} \mathrm{O}_{3}, \mathrm{SiO}_{2}, \mathrm{CaO}\right.$, $\mathrm{MgO}$ ). Normal muonium can coexist with shallow states when the gap lies between 5 and $7 \mathrm{eV}\left(\mathrm{Li}_{2} \mathrm{O}, \mathrm{Y}_{2} \mathrm{O}_{3}, \mathrm{HfO}_{2}\right.$, $\mathrm{SrO}, \mathrm{ZrO}_{2}, \mathrm{YSZ}$, and maybe also $\mathrm{La}_{2} \mathrm{O}_{3}$ ). Shallow-donor states predominate for gaps below $5 \mathrm{eV}\left(\mathrm{BaO}, \mathrm{CeO}_{2}, \mathrm{TiO}_{2}\right.$, $\mathrm{ZnO}, \mathrm{SnO}_{2}, \mathrm{WO}_{3}, \mathrm{CdO}$, maybe also $\mathrm{Ta}_{2} \mathrm{O}_{5}$ and $\mathrm{Nb}_{2} \mathrm{O}_{5}$ ).

The correlation can be understood in terms of the energy balance between promotion of the electron to the conduction band, $\mathrm{H}^{0} \rightarrow \mathrm{H}^{+}+\mathrm{e}_{\mathrm{c}}$, and stabilization of the proton by hydroxide-ion formation [23], $\mathrm{H}^{+}+\mathrm{O}^{2^{-}} \rightarrow \mathrm{OH}^{-}$. Some exceptions occur in semiconducting oxides with gaps below $2.5 \mathrm{eV}\left(\mathrm{Cu}_{2} \mathrm{O}, \mathrm{Ag}_{2} \mathrm{O}, \mathrm{Bi}_{2} \mathrm{O}_{3}\right.$ and $\left.\mathrm{HgO}\right)$, where other criteria evidently apply. Including also consideration of $\mathrm{H}^{-}$, in a scheme we call the 3-Delta model, the implications for hydrogen doping may be similarly described [15,22].

\footnotetext{
${ }^{2}$ Fitting procedures for repolarization and resonance data involving state conversion and other dynamics are described in an accompanying paper [21].
} 
Observation of the shallow-donor state is a necessary condition for the material to exhibit hydrogen-induced electronic conductivity but it is not sufficient: $\mathrm{H}$ may be self-compensating if it also exhibits a deep acceptor level. So far, identification of the acceptor levels and observation of the negative ion remain elusive.

\section{References}

[1] E.V. Minaichev, et al., Sov. Phys. JETP 31 (1970) 849.

[2] J.H. Brewer, et al., Phys. Rev. Lett. 31 (1973) 143.

[3] J.H. Brewer, Oral presentation at Yamada Conference VII, Shimoda, 1983, unpublished.

[4] D.P. Spencer, et al., Hyperfine Interactions 17-19 (1984) 567.

[5] P.W. Percival, et al., Chem. Phys. 95 (1985) 321.

[6] R.F. Kiefl, et al., Phys. Rev. B 34 (1986) 1474.

[7] V.N. Duginov, et al., Hyperfine Interactions 85 (1994) 197.
[8] A. Amato, et al., PSI Scientific Rep. III (2002) 123.

[9] J.W. Schneider, et al., Phys. Rev. B 41 (1990) 7254.

[10] W.K. Dawson, et al., Hyperfine Interactions 106 (1997) 97.

[11] S.F.J. Cox, et al., Phys. Rev. Lett. 86 (2001) 2601.

[12] K. Shimomura, et al., Phys. Rev. Lett. 89 (2002) 255505.

[13] M. Charlton, et al., ISIS Annual Report (2000) Expt. RB11350.

[14] K. Shimomura, et al., Physica B (ICDS 2005), in press.

[15] S.F.J. Cox, et al., J. Phys.: Condens. Matter, in press.

[16] J.S. Lord, et al., J. Phys.: Condens. Matter (special MuSSR issue) 16 (2004) S4707.

[17] J.S. Lord, et al., Physica B (Paper ID 40/P71).

[18] S.F.J. Cox, et al., J. Phys.: Condens. Matter 13 (2001) 9000.

[19] J. Piroto Duarte, et al., Physica B (Paper ID 185/P29).

[20] J.H. Brewer, (2002), private communication.

[21] J.S. Lord, Physica B (Paper ID 55/P74).

[22] S.F.J. Cox, et al., Physica B (Proc. ICDS 2005), in press, doi:10.1016/ j.physb.2005.12.099.

[23] C. Boekema, et al., Hyperfine Interactions 8 (1981) 609. 